\title{
Near-complete recanalization of jugular vein and multiple dural sinus thromboses with warfarin in a case of antiphospholipid syndrome
}

\author{
Ufuk Ilgen, Sezin Turan, Hakan Emmungil \\ Department of Rheumatology, Trakya University School of Medicine, Edirne, Turkey
}

\begin{abstract}
Antiphospholipid syndrome is a cause of arterial and venous thrombosis especially in the young adult population. Although dural sinus thrombosis is a relatively rare complication of antiphospholipid syndrome, it may be a cause of morbidity and mortality. Extension of thrombosis and involvement of deep venous structures are poor prognostic factors in patients with dural sinus thrombosis, but the rate of near-complete recanalization is not known in antiphospholipid syndrome-related dural sinus thrombosis. Herein, a case of antiphospholipid syndrome with multiple dural sinus, deep cerebral vein and internal jugular vein thromboses is presented with demonstrative imaging findings and near-complete recanalization after warfarin.
\end{abstract}

Key words: antiphospholipid syndrome, dural sinus thrombosis, warfarin.

\section{Introduction}

Antiphospholipid syndrome (APS) is responsible for about $10 \%$ of hypercoagulable states in the young adult population [1]. In a large multinational observational study, APS was found to constitute $5.9 \%$ of cases of dural sinus thrombosis [2]. Although dural sinus thrombosis is a relatively rare complication of antiphospholipid syndrome, it is a potential cause of morbidity and mortality. Extension of thrombosis and involvement of deep venous structures are poor prognostic factors and are associated with complications such as venous infarction, epilepsy, and increased intracranial pressure in patients with dural sinus thrombosis, but the rate of near-complete recanalization is not known in APS-related dural sinus thrombosis [2, 3].

Warfarin was shown to be of benefit both in secondary prophylaxis in patients with APS and in the management of patients with dural sinus thrombosis $[4,5]$.

\section{Case report}

A 38-year-old woman had sudden onset severe headache with no other sign of increased intracranial pressure. Vital signs, systemic and neurological examinations were normal. Intracranial hemorrhage was excluded with computed tomography. Cranial magnetic resonance $(M R)$ imaging was suggestive of dural sinus thrombosis and MR venography revealed total thrombosis in the superior sagittal, right transverse, right sigmoid, straight sinuses, and the great cerebral and right internal jugular veins (Fig. 1 A, B).

The distal part of the left transverse sinus was also totally thrombosed. Treatment with warfarin was started after an initial course of heparin. Tests for genetic thrombophilia were negative with no history of smoking or oral contraceptive use. The patient had a high titer of serum anti- $\beta 2$-glycoprotein I lgG (87.2 GPL U/ml) and moderately elevated anticardiolipin in IgG class gamma globulins (32.5 GPL U/ml). Lupus anticoagulant (LAC) was negative and serum anticardiolipin in IgM class and anti$\beta 2$-glycoprotein I IgM were normal. In conclusion, the patient presents double positivity in antiphospholipid antibodies. Complete blood count, serum biochemistry and urinary examination were normal. No symptom, sign or immunological feature suggesting systemic lupus erythematosus was present. After five months of

Address for correspondence:

Ufuk Ilgen, Department of Rheumatology, Trakya University School of Medicine, 22100 Edirne, Turkey, e-mail: ufukilgen@gmail.com

Submitted: 24.09.2018; Accepted: 23.11.2018 

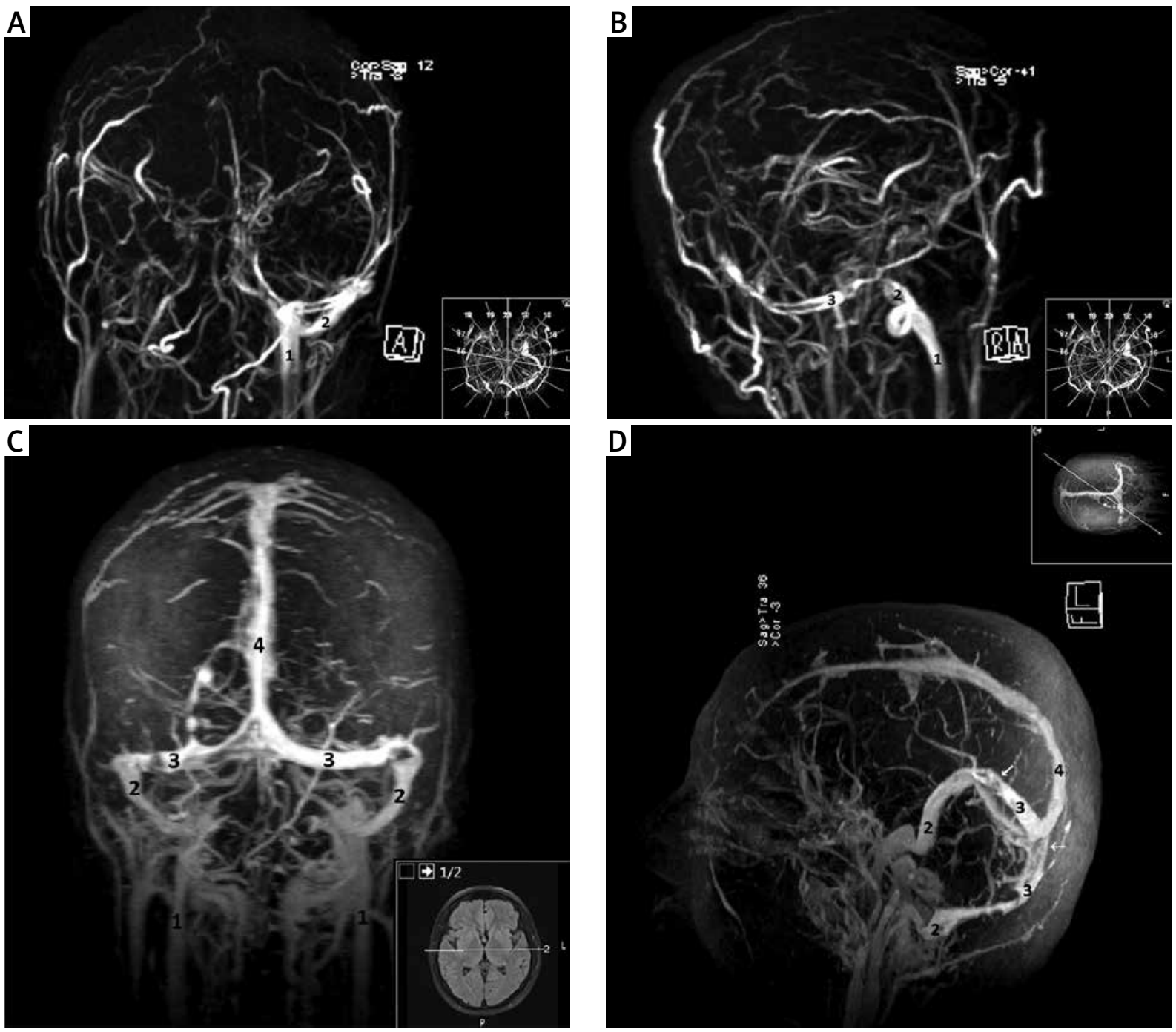

Fig. 1. Initial (A, B) and control (C, D) magnetic resonance venography. Initially, the left transverse sinus has luminal signal loss indicating thrombosis and continuity with the left sigmoid sinus is not observed (B). Note that the superior sagittal, straight, right transverse, right sigmoid sinuses, and the great cerebral and right internal jugular veins are not observed at all. After treatment with warfarin, note near-complete opening of initially thrombotic sinuses and veins. Loss of signals in transverse sinuses and confluence of sinuses indicate chronic partial thromboses (arrows). 1 - internal jugular veins, 2 - sigmoid sinuses, 3 - transverse sinuses, 4 - superior sagittal sinus.

treatment with warfarin with an international normalized ratio (INR) of 2.5-3, control MR venography revealed near-complete recanalization of dural sinuses and the right internal jugular vein (Fig. 1 C, D).

High titer anti- $\beta 2$-glycoprotein I IgG and moderately elevated anticardiolipin IgG persisted (85 and $31 \mathrm{GPL}$ $\mathrm{U} / \mathrm{ml}$, respectively) five months after the first measurement and antiphospholipid syndrome was diagnosed. No history of pregnancy was present. She is still being followed up under warfarin without any symptoms.

\section{Discussion}

Near-complete recanalization of such an extensive cerebral venous thrombosis is thought to be rare, especially when accompanied by a systemic thrombotic disorder and poor prognostic features, although no cohort of dural sinus thrombosis or APS-related thrombosis has been assessed with respect to this issue to date. In previous studies researchers found that double positivity, which means $\beta 2-G P I$ antibodies and LAC presence, is 
a triggering factor of arterial thrombosis; therefore low positivity may cause venous thrombosis $[6,7]$. However, the described patient had $\mathrm{aCL}$ and $\beta 2$-GPI antibodies. Recently it has been highlighted that the positivity to IgA anti- $\beta 2 \mathrm{GPI}$ antibody was clinically relevant in APS patients [8], although the association between thrombosis and $\mathrm{aCL}$ or $\mathrm{a} \beta 2-\mathrm{GPI}$ is not so obvious as between LAC or triple positivity ( $\mathrm{aCL}+\mathrm{LAC}+\mathrm{a} \beta 2-\mathrm{GPI}$ antibodies) $[8,9]$.

The APS diagnosis is based on the new Sydney criteria, which suggest performing laboratory tests at least 12 weeks after the clinical symptoms (thrombosis), and also the time interval cannot be more than 5 years between the clinical symptoms and the test to make the diagnosis of APS [10].

It is a matter of debate whether a relatively high INR, unintentionally kept between 2.5 and 3 in the present case, contributed to near-complete resolution of thrombosis, since high-intensity anticoagulation with warfarin (INR 3-4) was not shown to be superior to moderate intensity anticoagulation (INR 2-3) in terms of thromboprophylaxis [11].

\section{Conclusions}

The prognosis of APS-related dural sinus thrombosis is largely unknown. Anticoagulation with warfarin, shown to be effective both in secondary prevention in patients with APS and in the treatment of patients with dural sinus thrombosis $[4,5]$, is still the mainstay of management in APS-related dural sinus thrombosis, since new oral anticoagulants are not proven to be more effective or safer in APS [12].

\section{Acknowledgements}

Written informed consent was obtained from the patient.

The authors declare no conflict of interest.

\section{References}

1. Yokus O, Albayrak M, Balcik OS, et al. Risk factors for thrombophilia in young patients presenting with thrombosis. Int J H Hematol 2009; 90: 583-590.

2. Ferro JM, Canhao P, Stam J, et al. Prognosis of cerebral vein and dural sinus thrombosis: results of the International Study on Cerebral Vein and Dural Sinus Thrombosis (ISCVT). Stroke 2004; 35: 664-670.

3. Aguiar de Sousa D, Lucas Neto L, Canhao P, et al. Recanalization in cerebral venous thrombosis: a systematic review and meta-analysis. Stroke 2018. doi: 10.1161/STROKEAHA.118.022129 [Epub ahead of print].

4. Khamashta MA, Cuadrado MJ, Mujic F, et al. The management of thrombosis in antiphospholipid antibody syndrome. N Engl J Med 1995; 332: 993-997.

5. Patel SI, Obeid H, Matti L, et al. Cerebral venous thrombosis: current and newer anticoagulant treatment options. Neurologist 2015; 20: 80-88.

6. Reynaud Q, Lega JC, Mismetti P, et al. Risk of venous and arterial thrombosis according to type of antiphospholipid antibodies in adults without systemic lupus erythematosus: a systematic review and meta-analysis. Autoimmun Rev 2014; 13: 595-608.

7. Nojima J, Suehisa E, Akita N, et al. Risk of arterial thrombosis in patients with anticardiolipin antibodies and lupus anticoagulant. Br J Haematol 1997; 96: 447-450.

8. Andreoli L, Fredi M, Nalli C, et al. Clinical significance of IgA anti-cardiolipin and IgA anti- $\beta 2$ glycoprotein I antibodies. Curr Rheumatol Rep 2013; 15: 343.

9. Devreese KM. Antiphospholipid antibodies: evaluation of the thrombotic risk. Thromb Res 2012; 130 (Suppl 1): S37-40.

10. Miyakis S, Lockshin MD, Atsumi T, et al. International consensus statement on an update of the classification criteria for definite antiphospholipid syndrome (APS). J Thromb Haemost 2006; 4: 295-306.

11. Crowther MA, Ginsberg JS, Julian J, et al. A comparison of two intensities of warfarin for the prevention of recurrent thrombosis in patients with the antiphospholipid antibody syndrome. N Engl J Med 2003; 349: 1133-1138.

12. Sciascia S, Lopez-Pedrera C, Cecchi I, et al. Non-vitamin K antagonist oral anticoagulants and antiphospholipid syndrome. Rheumatology 2016; 55: 1726-1735. 